\title{
Termine
}

http://doi.org/10.1515/bd-2018-0056

In der folgenden Terminzusammenstellung werden Fortbildungsseminare und ähnliche Fachveranstaltungen nachgewiesen, die zumindest einer eingeschränkten Öffentlichkeit zugänglich sind, außerdem Jahrestagungen und vergleichbare Anlässe umfassenderer Art. Hingegen können Termine nicht berücksichtigt werden, die ausschließlich lokaler Art oder für einen internen Kreis von Bedeutung sind, zumal in diesem Falle bei den Beteiligten in der Regel die Kenntnis des Termins vorausgesetzt werden kann. Soweit an anderer Stelle im Bibliotheksdienst nähere Informationen über einen bestimmten Termin abgedruckt sind, wird darauf verwiesen. Erstmalig aufgenommene Termine sind mit einem * gekennzeichnet.

\section{Juni 2018}

4. „Über das Buch hinaus!“ Bücher mit digitaler Erweiterung, Lüneburg (s. Heft 3-4, S. 319)

5. „Über das Buch hinaus!“ Bücher mit digitaler Erweiterung, Hildesheim (s. Heft 3-4, S. 319)

6. Über das Buch hinaus!“ Bücher mit digitaler Erweiterung, Ganderkesee (s. Heft 3-4, S. 320)

6. Kreistreffen der Öffentlichen Bibliotheken und Landkreise. Kyffhäuserkreis, Bad Frankenhausen (s. Heft 2, S. 151)

7. Kundenorientierte Kommunikation - den passenden Ton finden, auch in stressigen Situationen, Berlin (s. Heft 3-4, S. 327)

9. Basiskurse für ehren- und nebenamtlich tätige Büchereileiter/innen und -mitarbeiter/innen 2017/2018 Teil 4, Neustadt/Weinstraße (s. Heft 8, S. 729)

11. Kinderbuch-Klassiker: Warum wir sie lieben und wie auch Kids von heute Freude daran haben, Lüneburg (s. Heft 3-4, S. 321)

12. Kinderbuch-Klassiker: Warum wir sie lieben und wie auch Kids von heute Freude daran haben, Hildesheim (s. Heft 3-4, S. 321)

12. Basiskurse für ehren- und nebenamtlich tätige Büchereileiter/innen und -mitarbeiter/innen 2017/2018 Teil 4, Koblenz (s. Heft 8, S. 729)

13. allegro-OEB Workshop: allegro meets Excel, Lüneburg (s. Heft 3-4, S.321)

18. Digitale Medien kompetent nutzen: Workshop, Oldenburg (s. Heft 3-4, S. 322) 
18.-19. Practice your English - Workshop, Berlin (s. Heft 3-4, S. 328)

19. Digitale Medien kompetent nutzen: Workshop, Hildesheim (s. Heft 3-4, S. 322)

20. Digitale Medien kompetent nutzen: Workshop, Lüneburg (s. Heft 3-4, S. 322)

20. Neue Wege des Bestandsmanagements, Erfurt (s. Heft 2, S. 147)

25. Einwerbung von Fördermitteln für Bibliotheken, Lüneburg (s. Heft 3-4, S. 323)

25. Interkulturelle Kompetenz in der Bibliothek stärken, Berlin (s. Heft 3-4, S. 328)

27. Kreistreffen der Öffentlichen Bibliotheken und Landkreise. UnstrutHainich-Kreis, Mühlhausen (s. Heft 2, S. 151)

\section{September 2018}

6.-7. Wie vermitteln wir Informationskompetenz? Didaktische Kompetenzen für die Vermittlung von Informationskompetenz, Berlin (s. Heft 3-4, S. 329)

10.-11. Von der Präsenzschulung zu E-Tutorial. Mediendidaktische Ansätze für IK-Angebote (s. Heft 3-4, S. 329)

12. Qualitätsmanagement - Fragen und Antworten, Erfurt (s. Heft 2, S. 147)

12. Webinare und Webkonferenzen durchführen und moderieren - ganz praktisch - Workshop, Berlin (s. Heft 3-4, S. 330)

19. Kreistreffen der Öffentlichen Bibliotheken und Landkreise. Wartburgkreis, Creuzburg (s. Heft 2, S. 151)

26. Kanban für Bibliotheken - Den Workflow in Teams verbessern! Berlin (s. Heft 3-4, S. 330)

26. Allegro-Anwendertreffen, Erfurt (s. Heft 2, S. 148)

\section{Oktober 2018}

16. RDA-Einführung Kompakt, Berlin (s. Heft 3-4, S. 331)

17.-18. RDA Aufbau- und Auffrischungskurs Modul 3 - Einfache/mittlere Monografien und monografische Reihen (s. Heft 3-4, S. 331)

18. Wissensmanagement in Bibliotheken - Am Beispiel der Stadtbücherei Würzburg, Berlin (s. Heft 3-4, S. 332)

18.-19. Verbesserungen mit kleinem Budget, Berlin (s. Heft 3-4, S. 332) 


\section{November 2018}

12.-13. Hybride Präsentation und analoger und digitaler Bestände und Services in Bibliotheken, Berlin (s. Heft 3-4, S. 333)

14. Kreistreffen der Öffentlichen Bibliotheken und Landkreise. Altenburg, Schmölln (s. Heft 2, S. 151)

21. Kreistreffen der Öffentlichen Bibliotheken und Landkreise. Saale-OrlaKreis, Pößneck (s. Heft 2, S. 152)

22. E-Books in Wissenschaftlichen Bibliotheken - erwerben, erschließen, präsentieren, Berlin (s. Heft 3-4, S. 333)

28. Basiswissen Gestaltung - Flyer, Plakate und Jahresberichte, Erfurt (s. Heft 2, S. 149)

\section{Dezember 2018}

5. Fachtagung für Bibliotheksleiter/Innen in Öffentlichen Bibliotheken, Erfurt (s. Heft 2, S. 149)

12. Kreistreffen der Öffentlichen Bibliotheken und Landkreise. Sonneberg und Hildburghausen, Sonneberg (s. Heft 2, S. 152) 